\title{
Évaluation des contraintes résiduelles dans les pièces injectées en thermoplastique renforcé de fibres courtes
}

\author{
Thomas Giroud et Michel Vincenta \\ Centre de Mise en Forme des Matériaux, École des mines de Paris, UMR CNRS 7635, BP 207, \\ 06904 Sophia Antipolis Cedex, France
}

Reçu le 10 juin 2003, accepté le 7 novembre 2003

\begin{abstract}
Résumé - Dans un premier temps, les phénomènes à l'origine des contraintes résiduelles en injection de thermoplastique sont décrits, avec les spécificités liées aux polymères renforcés de fibres. Le refroidissement hétérogène associé à une variation de comportement mécanique conduit aux contraintes d'origine thermique, auxquelles il faut ajouter les contraintes dues à la pression dans la phase fluide figée par la solidification. Les fibres de renfort introduisent une hétérogénéité et une anisotropie de comportement mécanique et de retrait. Dans un deuxième temps, une méthode de mesure des contraintes résiduelles basée sur l'enlèvement de couches et la mesure des moments de flexion est présentée. Elle permet la mesure des contraintes résiduelles pour des matériaux anisotropes comme les thermoplastiques renforcés de fibres. En général, on obtient des contraintes de traction en cœur, et de compression en peau. L'effet du fraisage pour enlever les couches est évalué. La pression et le temps de maintien, la température de régulation du moule, le taux de fibres influencent les profils de contraintes.
\end{abstract}

Mots clés : Injection / thermoplastiques / contraintes résiduelles / fibres

\begin{abstract}
Evaluation of residual stresses in short fiber reinforced thermoplastic molded parts. First, phenomena at the origin of residual stresses in injection molding of thermoplastic polymers are described, and the specificity of fiber reinforced thermoplastic is introduced. Heterogeneous cooling associated with mechanical behavior variations leads to thermal stresses. Frozen-in pressure induced stresses are specific to the injection molding process. Fibers induce heterogeneity and anisotropy of mechanical behavior and thermal shrinkage. Second, a measurement technique based on the layer removal method and measurement of the bending moment is presented. It allows the determination of residual stresses in anisotropic materials such as fiber reinforced thermoplastics. The influence of the milling process is discussed. Tensile stresses in the core and compression stresses in the surface are usually obtained. Packing pressure and time, mold temperature, fiber concentration have an influence on the stress profiles.
\end{abstract}

Key words: Injection molding / thermoplastic / residual stresses / fibers

\section{Introduction}

La déformation des pièces en sortie de moule est un des problèmes les plus difficile à résoudre pour les concepteurs d'outillage d'injection de thermoplastiques. Ces déformations sont liées aux contraintes résiduelles. Si une dissymétrie existe dans l'épaisseur de la pièce, alors elle se voile au démoulage. Les contraintes résiduelles affectent également les propriétés mécaniques et optiques des pièces moulées. Elles peuvent avoir deux origines :

- les contraintes dues à l'écoulement pendant le remplissage et le compactage qui ne se relaxent pas

\footnotetext{
a Auteur correspondant : michel.vincent@ensmp.fr
}

complètement au moment de la solidification du fait du caractère viscoélastique du matériau. On parle de contraintes figées induites par l'écoulement;

- le retrait thermique associé au refroidissement hétérogène, qui conduit aussi à des contraintes résiduelles appelées contraintes thermiques. On les retrouve dans bon nombre de procédés et de matériaux, comme le verre et les métaux.

Lorsque le polymère est renforcé de fibres, le retrait et le comportement à l'état solide deviennent fortement anisotropes. En effet, les fibres sont orientées en fonction de l'écoulement, et le retrait des fibres de verre et du polymère sont très différents. Les phénomènes de voilage 
des pièces sont souvent encore plus problématiques que pour les polymères non renforcés.

Dans un premier temps, nous montrerons les mécanismes mis en jeu, avec les spécificités liées aux fibres de renfort. Dans un deuxième temps, nous présenterons une méthode de mesure adaptée à des matériaux hétérogènes, et les profils de contraintes dans différentes conditions d'injection.

\section{Les phénomènes physiques principaux}

Nous allons d'abord présenter les phénomènes généraux intervenant lors de l'injection des thermoplastiques, puis les particularités liées à la présence des fibres de renfort.

\subsection{Le retrait thermique}

\subsubsection{Trempe libre}

C'est l'effet le plus largement cité. Supposons qu'en dessous d'une température de solidification $T_{\mathrm{s}}$ le polymère se comporte comme un solide élastique, et au-dessus, comme un liquide, qui ne gêne pas le retrait. Considérons une plaque plane. Deux couches se solidifient d'abord en surface au contact du moule froid. Elles sont libres d'effectuer leur retrait thermique, car le cour est encore en phase liquide, et accommode la déformation. La déformation associée est $\alpha\left(T_{\mathrm{s}}-T_{\mathrm{f}}\right)$, où $\alpha$ est le coefficient de dilatation linéaire et $T_{\mathrm{f}}$ la température finale. Les couches plus à l'intérieur vont se solidifier plus tard, elles seront soumises à la même déformation $\alpha\left(T_{\mathrm{s}}-T_{\mathrm{f}}\right)$, mais leur retrait sera bloqué par les couches externes. Elles se retrouveront donc en traction, et mettront les couches externes en compression.

\subsubsection{Refroidissement dans le moule}

Considérons maintenant la pièce dans le moule. Si on suppose que le contact polymère-cavité est collant, tant que la pièce est dans le moule, aucun retrait ne peut s'effectuer lorsque la température d'une couche descend en dessous de la température de solidification, et cette couche se retrouve par conséquent en traction. Si le matériau se solidifie entièrement dans le moule, alors il se retrouve en traction uniforme, et au démoulage, ces contraintes se relâcheront. En revanche, si le démoulage a lieu alors que le polymère n'est pas solidifié sur toute son épaisseur, on aboutit à un état de traction/compression analogue à celui du refroidissement libre.

Ces analyses qualitatives sont à tempérer compte tenu des éléments suivants. D'abord, le polymère solide n'a pas un comportement purement élastique, mais viscoélastique. Comme les couches de polymère se solidifient à des instants différents, leurs histoires sont également différentes, et la relaxation des contraintes intervient de façon hétérogène. Ensuite, l'hypothèse de contact collant pièce-moule est probablement réaliste tant qu'une forte pression de maintien plaque les couches solides contre le moule, ou lorsque la géométrie de l'empreinte « bloque » la pièce (noyau, insert, courbure). Dans d'autres cas, il pourra y avoir glissement.

\subsection{Les contraintes figées}

L'écoulement, et les contraintes qui en résultent, ont un effet sur l'orientation moléculaire et des fibres, sur la cristallisation. Elles induisent une anisotropie et une hétérogénéité de comportement rhéologique à l'état solide et de retrait. Les contraintes de cisaillement figées sont réputées d'un ordre de grandeur inférieur aux autres sources de contraintes [1].

En revanche, la pression figée est primordiale [2-5]. $\mathrm{Au}$ cours du cycle d'injection, les différentes couches se solidifient à des niveaux de pression différents, par exemple relativement faibles en peau pendant le remplissage, élevés sous la peau pendant le compactage, moyen à cœur lorsque le seuil est solidifié et que la pression imposée par la machine n'est plus transmise. Lorsque la pièce sort du moule, chaque couche subit une expansion liée au retour à la pression atmosphérique, d'autant plus élevée que la pression figée est grande. Comme les couches sont solidaires, il y a une expansion moyenne, et on obtient le plus souvent des contraintes de traction à cœur, de compression en peau, et éventuellement de traction en extrême peau.

\subsection{Cas du refroidissement dissymétrique}

Il est fréquent d'avoir un transfert thermique différent sur les deux faces du moule, souvent involontairement. On a toutes les chances d'avoir une géométrie de pièce fort différente de celle de l'empreinte.

Dans le cas d'un refroidissement libre, on montre que le maximum de contrainte se déplace du côté refroidi le moins efficacement [6] (Fig. 1). La pièce se courbe vers ce même côté.

En injection, il faut ajouter la contribution de la pression figée. Il est plus difficile de conclure, et la courbure pourra se faire soit du côté chaud soit du côté froid selon la cinétique de solidification et l'évolution de pression figée.

\subsection{Les spécificités des pièces injectées avec un thermoplastique renforcé de fibres}

L'orientation des fibres induites par l'écoulement, en plus de l'orientation moléculaire, entraîne une anisotropie de comportement rhéologique et de coefficient de dilatation.

La figure 2 représente l'évolution du module d'élasticité d'un polyamide renforcé à $30 \%$ en poids de fibres courtes, dans un échantillon dont les fibres sont orientées de façon unidirectionnelle, en fonction de l'angle 


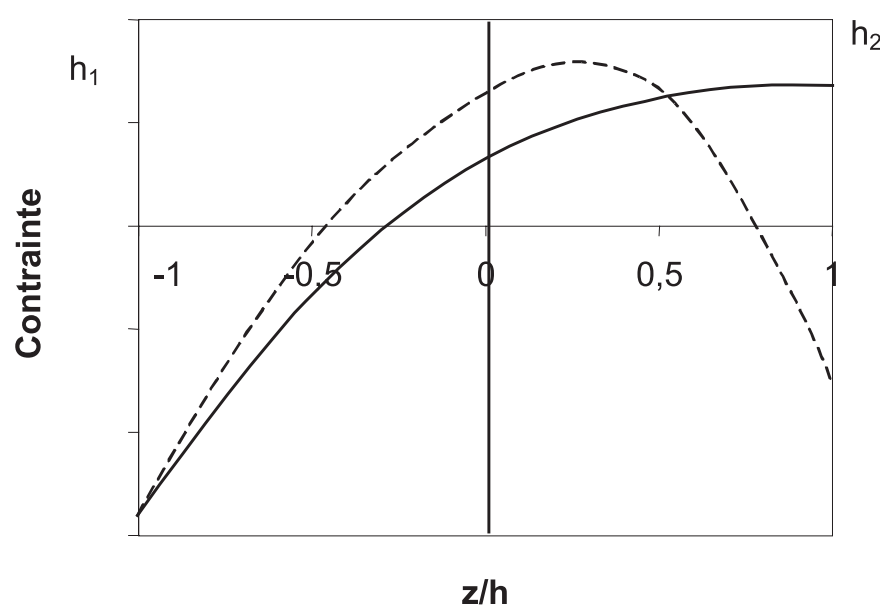

Fig. 1. Cas d'un refroidissement avec des coefficients de transfert thermique $h_{1}$ et $h_{2}$ différents sur les deux faces : $-: h_{2}$ pour $\mathrm{z} / \mathrm{h}=1$ est plus faible que $\mathrm{h}_{1}$ pour $\mathrm{z} / \mathrm{h}=-1,-\mathrm{h}_{2}$ est nul.

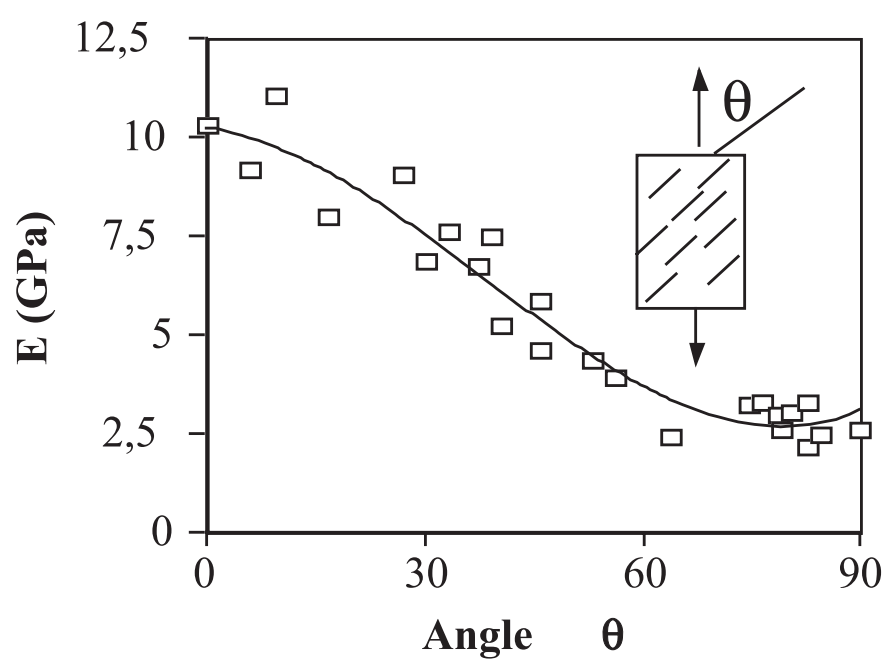

Fig. 2. Évolution du module d'élasticité d'un polyamide 66 renforcé à $30 \%$ en poids de fibres de verre courtes en fonction de l'angle entre les fibres et la direction de traction.

entre les fibres et la direction de traction. On observe un facteur de l'ordre de 4 entre le module maximal et le module minimal. On a également des variations significatives du coefficient de dilatation linéaire, dont vont dépendre les contraintes résiduelles d'origine thermique, en fonction de l'orientation des fibres, en raison de la différence entre le coefficient du verre, de l'ordre de $5 \times 10^{-6}{ }^{\circ} \mathrm{C}^{-1}$, et celui du polymère, de l'ordre de $7 \times 10^{-5}{ }^{\circ} \mathrm{C}^{-1}$.

L'anisotropie résultante dépendra donc de l'orientation des fibres. On observe souvent dans les pièces injectées une orientation de fibres dans la direction locale de l'écoulement en surface, et perpendiculaire à cour. Ceci est dû à la cinématique de l'écoulement [7]. Les écoulements de cisaillement induisent une orientation préférentielle dans la direction de l'écoulement, mais pas parfaite, car les fibres ne tendent pas vers une position d'équilibre. En élongation, les fibres s'orientent parallèlement à la direction de l'écoulement (respectivement perpendiculairement) avec un taux d'élongation positif (respectivement négatif). Si les interactions entre fibres étaient négligeables, cette orientation serait stable.

L'orientation dépendant de l'épaisseur et de la position dans la pièce, le retrait thermique et le comportement rhéologique à l'état solide sont anisotropes et hétérogènes, ce qui a une incidence directe sur le voilage de la pièce et les contraintes résiduelles.

\section{Mesure des contraintes résiduelles}

\subsection{Principe de la méthode}

La méthode d'enlèvement de couche a été la plus utilisée pour les pièces en polymère. Elle a été introduite par Treuting et Read [8] pour des métaux. Un échantillon plan est extrait de la pièce (ce n'est évidement pas possible sur des pièces de forme complexe). Des couches de 0,01 à $0,1 \mathrm{~mm}$ sont progressivement retirées de cet échantillon. On mesure à chaque fois la courbure du reste de l'échantillon résultant du nouvel équilibre de contraintes, et une analyse élastique, isotrope et homogène permet d'obtenir la répartition de contraintes dans l'épaisseur. Cette méthode est délicate à mettre en œuvre, et son principe n'est pas exempt de critique. Il faut en effet faire attention aux perturbations apportées lors de l'enlèvement des couches (échauffement si le fraisage mécanique est choisi). Par ailleurs, le module varie dans la pièce, surtout si elle est renforcée de fibres, ce qui la rend inapplicable dans ce dernier cas.

Une variante de la méthode évitant cet écueil consiste à mesurer la force nécessaire pour remettre à plat l'échantillon après enlèvement de la couche [9]. On accède ainsi, sans avoir besoin du module élastique, à la contrainte qu'il y avait dans la couche enlevée. On montre que sous l'hypothèse des petites déformations, le moment de flexion $M_{x}$ est relié à la force $F$ et à la longueur $L$ de l'échantillon par :

$$
M_{x}=\frac{F L}{6}
$$

et la contrainte à la cote $z(z=0$ correspond au plan médian de l'échantillon, et $z=z_{0}$ correspond à la surface initiale) vaut :

$\sigma_{x}(z)=\frac{2}{\left(z_{0}+z\right)} \frac{\mathrm{d} M_{x}(z)}{\mathrm{d} z}+2 \frac{M_{x}(z)}{\left(z_{0}+z\right)^{2}}-4 \int_{z}^{z_{0}} \frac{M_{x}(\xi)}{\left(z_{0}+\xi\right)^{3}} \mathrm{~d} \xi$

Le dépouillement passe donc par un lissage de la courbe mesurée $M_{x}(z)$, pour déterminer les dérivées et intégrales présentes dans l'équation (2).

\subsection{Mise en place expérimentale}

Les couches ont été retirées par fraisage. L'échantillon est ensuite placé sur deux appuis, et un troisième appui est relié à la traverse d'une machine de traction pour appliquer une force au centre de l'éprouvette et remettre 


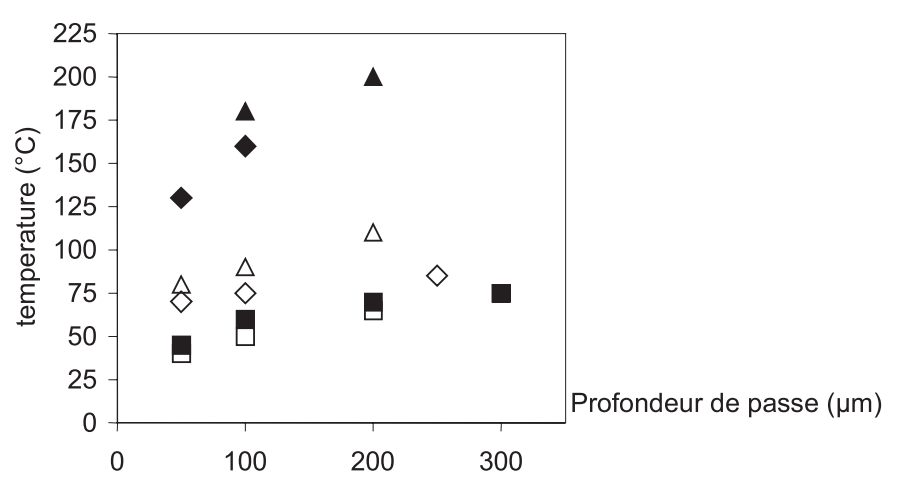

Fig. 3. Températures mesurées au niveau du contact fraisepolymère en fonction de l'épaisseur enlevée. Polypropylène, conditions C1 $(\square)$ et $\mathrm{C} 2(\mathbf{\square})$. PAA 50 , conditions C1 $(\diamond)$ et $\mathrm{C} 2(\diamond)$. PAA, conditions $\mathrm{C} 1(\triangle)$ et $\mathrm{C} 2(\boldsymbol{\Delta})$.

l'échantillon à plat. Cette force est mesurée avec un capteur de $2 \mathrm{~N}$, et la position de la traverse est contrôlée avec un capteur LVDT de 0,01 mm de précision.

L'effet du fraisage a été étudié en détail, en particulier l'échauffement du polymère au passage de la fraise. Nous avons testé deux conditions extrêmes compte tenu de notre dispositif. Une condition douce $\mathrm{C} 1$, avec une fraise de $2 \mathrm{~mm}$ de diamètre tournant à 8000 tr. $\mathrm{min}^{-1}$, et une condition sévère $\mathrm{C} 2$ avec une fraise de $6 \mathrm{~mm}$ de diamètre tournant à $24000 \mathrm{tr} \cdot \mathrm{min}^{-1}$. La vitesse d'avance est dans les deux cas de $5 \mathrm{~mm} . \mathrm{s}^{-1}$.

Deux techniques de mesure de la température ont été utilisées. D'abord, une sonde à infra rouge suit la fraise au niveau du contact. La figure 3 montre l'évolution de la température avec l'épaisseur de polymère enlevé, pour un polypropylène (Eltex HV252 de Solvay), un polyarylamide, sans fibre (noté PAA) et avec $50 \%$ en poids de fibres de verre courtes (IXEF 1022 de Solvay, noté PAA 50) de longueur moyenne d'environ $150 \mu \mathrm{m}$ et de diamètre $10 \mu \mathrm{m}$.

Ensuite, un thermocouple de $0,5 \mathrm{~mm}$ de diamètre est implanté dans l'échantillon, et on mesure la température au fur et à mesure des passes. Les enregistrements montrent que l'échauffement est ressenti seulement lorsque la fraise est proche de la verticale du thermocouple. Il est important, de l'ordre de $40{ }^{\circ} \mathrm{C}$ pour le polypropylène, et de 100 à $160{ }^{\circ} \mathrm{C}$ pour le polyarylamide. Il se produit lorsque la distance fraise-thermocouple est faible, de l'ordre de 100 à $500 \mu \mathrm{m}$ selon les matériaux, comme le montre la figure 4.

Les températures mesurées restent en dessous des températures de fusion des différents matériaux. Cependant, les résidus de coupe semblent avoir fondu, puisqu'ils ont dans certains cas plutôt une forme de bille que de copeau. Les températures que nous avons obtenues sont donc probablement des valeurs par défaut.

L'incidence des conditions de fraisage (diamètre de fraise, vitesse de rotation et d'avance, profondeur de passe) sur les contraintes mesurées a été déterminée avec les polypropylène et polyarylamide renforcé à $50 \%$ en fibres de verre. On obtient des profils et des niveaux de contrainte souvent très différents. L'usinage a donc

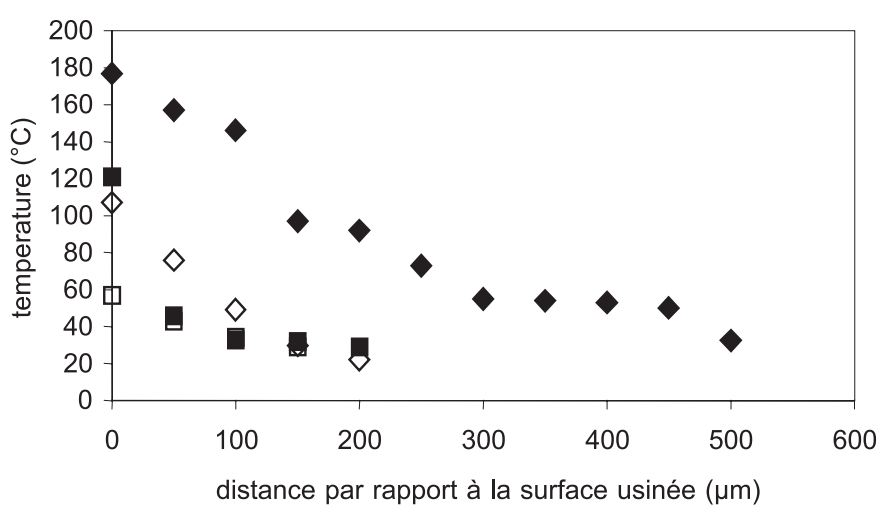

Fig. 4. Température maximale mesurée par le thermocouple en fonction de la distance entre celui-ci et la fraise. Polypropylène, conditions $\mathrm{C} 1(\square)$ et $\mathrm{C} 2(\boldsymbol{\square})$. PAA 50 , conditions $\mathrm{C} 1(\diamond)$ et $\mathrm{C} 2(\diamond)$.

une incidence sur la mesure. On montre cependant que pour certaines conditions, la variation de certains paramètres n'influence plus trop le profil final. Ainsi, pour un diamètre de fraise petit $(2 \mathrm{~mm})$ et pour une vitesse d'avance rapide $\left(100 \mathrm{~mm} . \mathrm{s}^{-1}\right)$, la vitesse de rotation de la fraise mais surtout la profondeur de passe n'influent pas sur les valeurs et la forme du profil, tant pour le polypropylène que pour le polyarylamide renforcé à $50 \%$. On privilégiera de plus une faible vitesse de rotation, pour minimiser l'échauffement. Nous établissons donc un protocole d'usinage «stable » : diamètre $2 \mathrm{~mm}$, profondeur de passe :

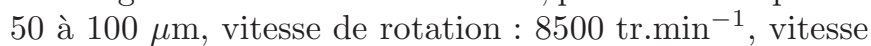
d'avance : $100 \mathrm{~mm} . \mathrm{s}^{-1}$, qui nous permet d'avoir des mesures bien reproductibles et de montrer l'influence des paramètres d'injection sur le profil de contraintes résiduelles final. Remarquons qu'il serait hasardeux de vouloir comparer différents polymères entre eux, étant données les différences de dégagement de chaleur observées pour une même condition d'usinage.

La question de savoir si l'enlèvement successif de couches a pour conséquence de relaxer l'état de contrainte initial ou, au contraire, de générer de nouvelles contraintes reste posée. On se retrouve, en effet, dans la situation d'un matériau dont l'une des surfaces est chauffée puis refroidie de nouveau (alors que l'autre reste froide) sans qu'aucun retrait ne puisse être effectué (l'échantillon est collé à la table par de l'adhésif double face). Cette situation est propice à la construction de contraintes résiduelles (thermiques) supplémentaires.

\subsection{Comparaison de la méthode de Treuting et Read, et de la méthode des moments de flexion}

Pour tester la validité de la technique, nous la comparons à la méthode de dépouillement traditionnelle basée sur la mesure de la courbure, avec des matériaux sans fibres. Avec un ABS (Fig. 5a), l'accord est bon. Ce matériau amorphe a en effet une structure relativement homogène. Avec le polypropylène (Fig. 5b), on constate des écarts, en particulier en surface. Ceci peut être dû aux 


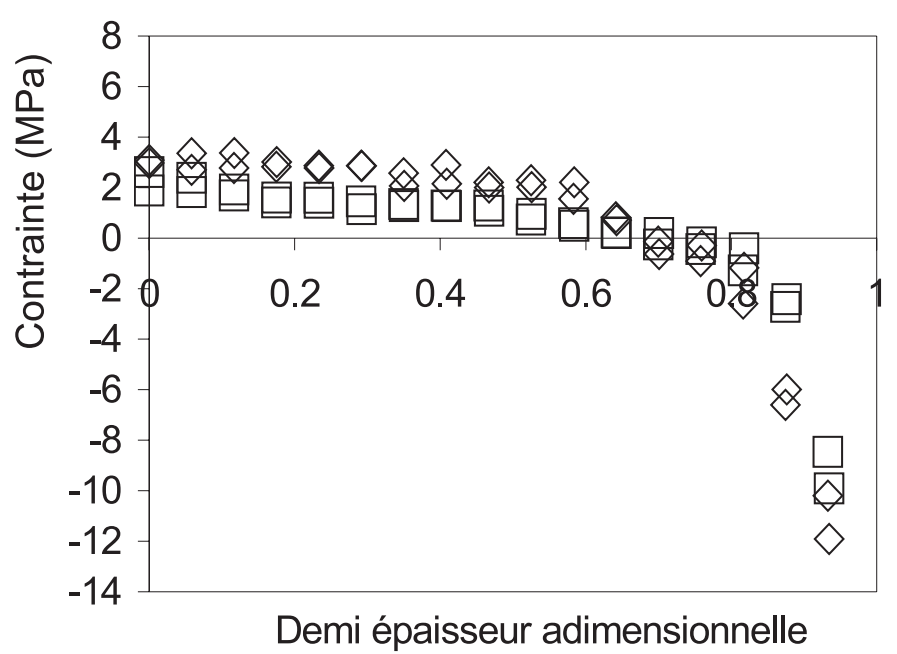

(a)

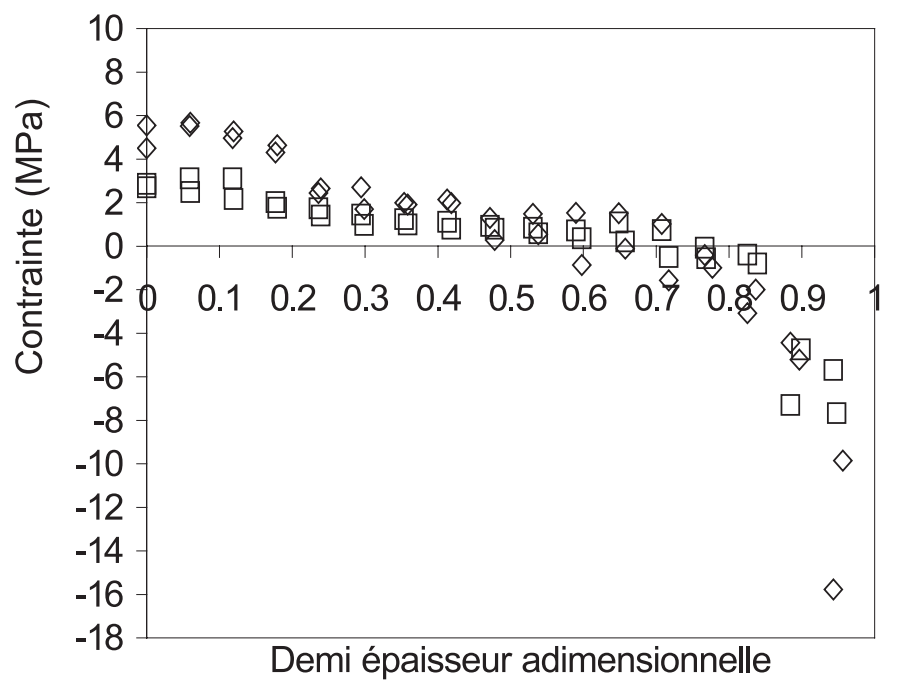

(b)

Fig. 5. Profils de contraintes mesurés dans la demi épaisseur d'une pièce par la technique des moments $(\square)$ et de Treuting et Read $(\diamond)$. (a) : ABS. (b) : polypropylène.

variations des propriétés élastiques dans l'épaisseur, associée à une structure semi-cristalline très différente entre la peau et le centre, qui mettent en défaut la technique de dépouillement classique. Sur la figure 5 sont superposées deux mesures sur deux échantillons différents injectés dans les mêmes conditions. On constate que la reproductibilité est correcte.

\subsection{Résultats}

On montre des résultats concernant une plaque de 200 par $40 \mathrm{~mm}$, de 1,7 mm d'épaisseur, en polyarylamide renforcé de $30 \%$ (IXEF 1002 de Solvay) ou $50 \%$ en poids de fibres de verre courtes, notés respectivement PAA30 et PAA50, en deux régions $\mathrm{P} 1$ et $\mathrm{P} 2$ situées près et loin de l'entrée (Fig. 6). Les échantillons extraits pour la mesure des contraintes font $60 \times 8 \mathrm{~mm}$. La figure 7 montre que

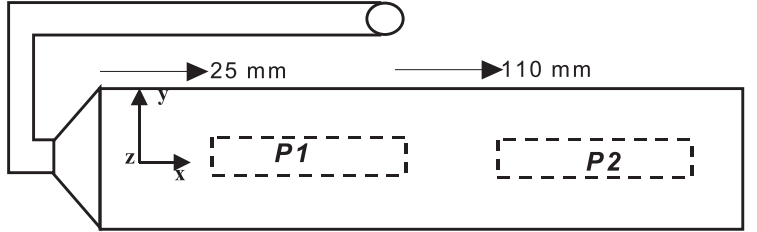

Fig. 6. Positions et tailles des échantillons extraits de la plaque injectée pour l'analyse des contraintes résiduelles.

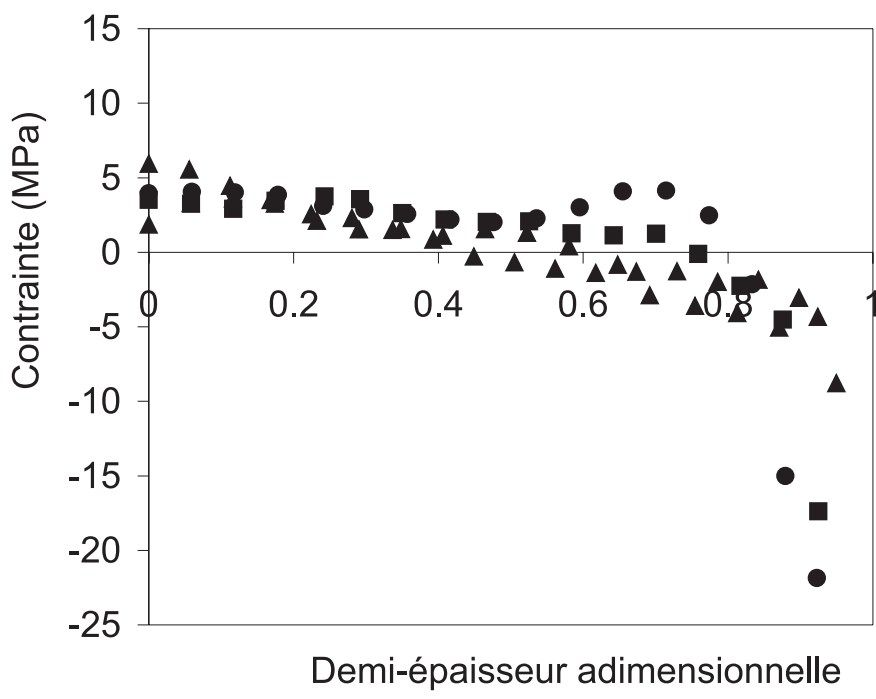

Fig. 7. Profil de contraintes sur PAA 50, en position P2. Incidence de la pression de maintien $15 \mathrm{MPa}(\bullet), 45 \mathrm{MPa}(\mathbf{\bullet})$, $85 \mathrm{MPa}(\mathbf{\Lambda})$.

quand la pression de compactage augmente, le niveau de contrainte diminue globalement, et en particulier en peau. Ceci est dû au phénomène de pression figée. La figure 8 montre que les tendances sont similaires lorsque le temps de maintien augmente.

La figure 9 compare les deux taux de renfort. Les différences sont significatives en peau, avec un niveau de contrainte plus élevé pour le PAA 50. Il est difficile d'interpréter ce résultat, car l'orientation des fibres est assez différente dans les deux échantillons. Celle-ci a été mesurée par microscopie optique sur des coupes polies [10]. On mesure les grand et petit axes de l'ellipse, coupe de la fibre circulaire avec le plan d'observation, d'où l'on déduit l'orientation dans l'espace. Les fibres sont en grande majorité parallèles au plan de la pièce. L'orientation dans ce plan est représentée par le paramètre $a_{x x}$, égal à la moyenne du carré du cosinus de l'angle que font les fibres avec la direction de l'écoulement $x$ (qui est aussi l'axe de la plaque). La figure 10 montre que l'orientation en peau pour les deux polymères est proche de la direction de l'écoulement, puisque $a_{x x}$ vaut environ 0,8 . ̊̀ coeur, les fibres du PAA 50 restent bien orientées dans cette même direction, tandis que celles du PAA 30 sont plutôt isotropes dans le plan (l'isotropie correspond à $\left.a_{x x}=0,5\right)$. Ces orientations, ainsi que le taux de renfort, modifient les propriétés mécaniques et de retrait, ainsi que la conductivité thermique. Il est encore difficile de 


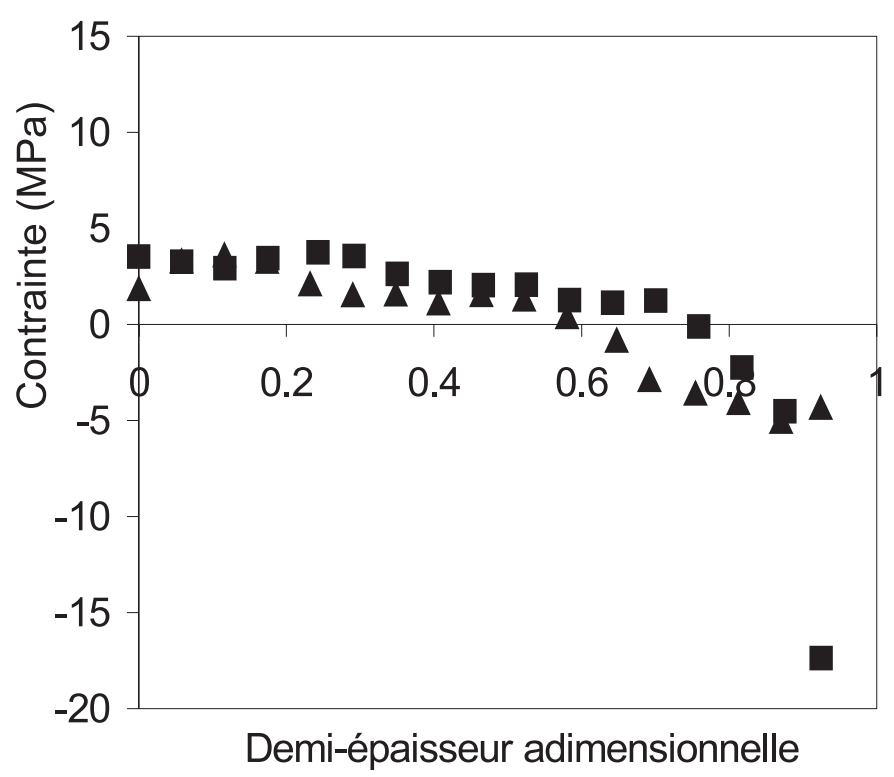

Fig. 8. Incidence du temps de maintien $15 \mathrm{~s}(\boldsymbol{\square}), 25 \mathrm{~s}(\boldsymbol{\Lambda})$ pour le PAA 50.

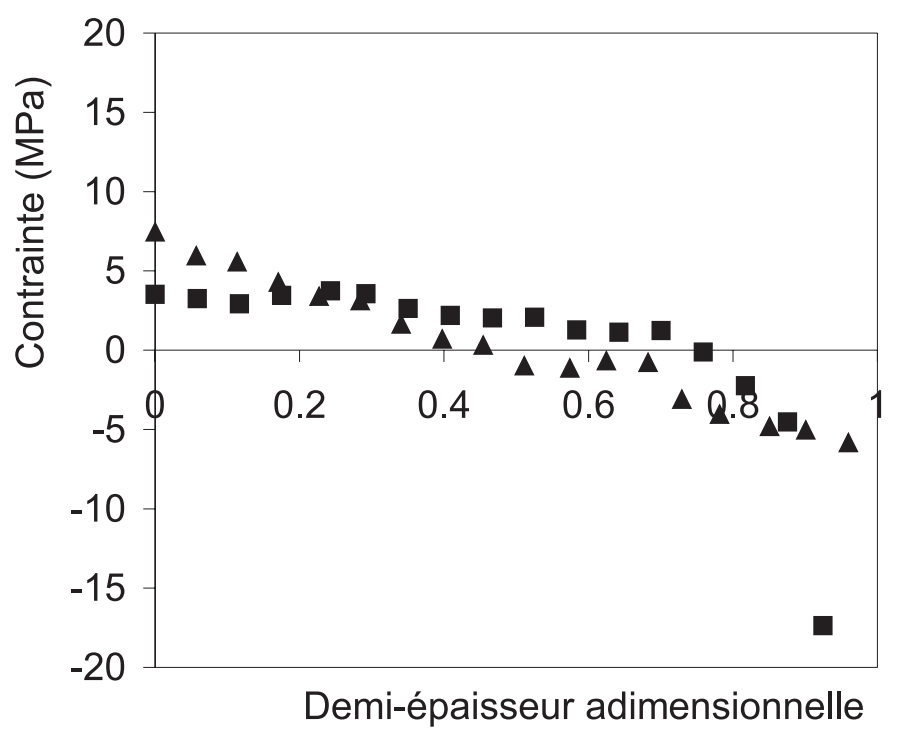

Fig. 9. Incidence du taux de fibres : PAA 30 (ム) et PAA $50(\mathbf{\square})$.

dire lequel de ces phénomènes est principalement responsable de la différence de contraintes résiduelles mesurée.

Enfin, la figure 11 montre les profils de contraintes résiduelles lorsque les deux faces du moule sont régulées à des températures différentes : $135^{\circ} \mathrm{C}$ et $150{ }^{\circ} \mathrm{C}$. Il est à noter que l'on détermine le profil de contraintes résiduelles sur deux demi-épaisseurs issues de deux échantillons différents, et on « recolle » les demi-profils. La continuité des mesures montre la qualité de la technique. L'asymétrie est beaucoup plus marquée en position P2. En P1, on remarque un état de tension en peau du côté chaud, que l'on peut attribuer à l'effet de la pression de maintien, plus active de ce côté puisque le refroidissement est moins efficace, et donc la solidification plus tardive. En P2, donc

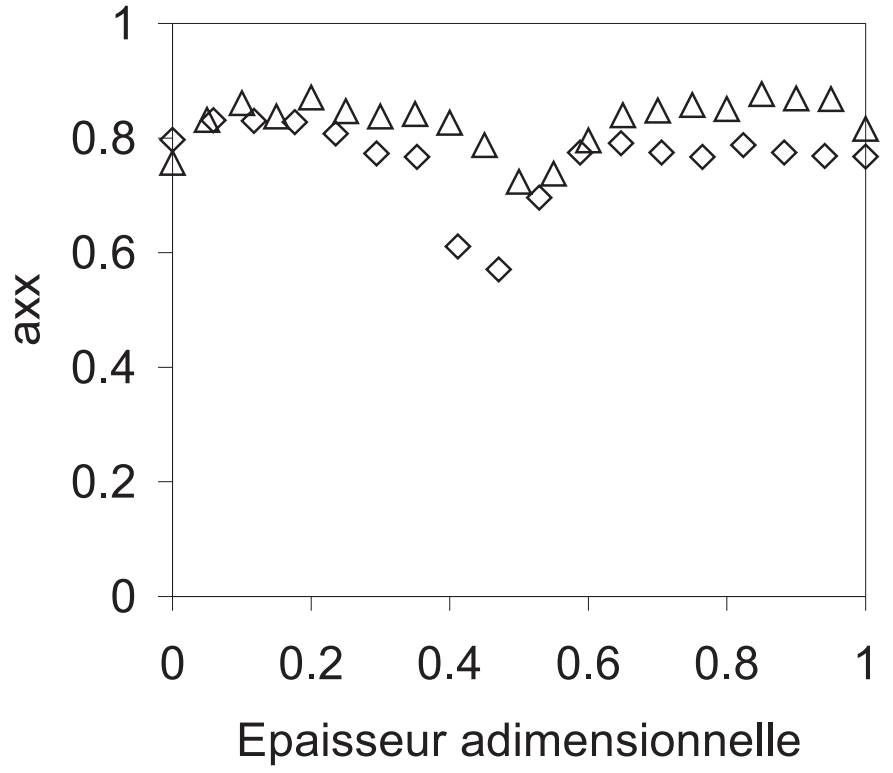

Fig. 10. Évolution de l'orientation dans l'épaisseur pour le PAA $50(\triangle)$ et le PAA $30(\diamond)$ en position P2.

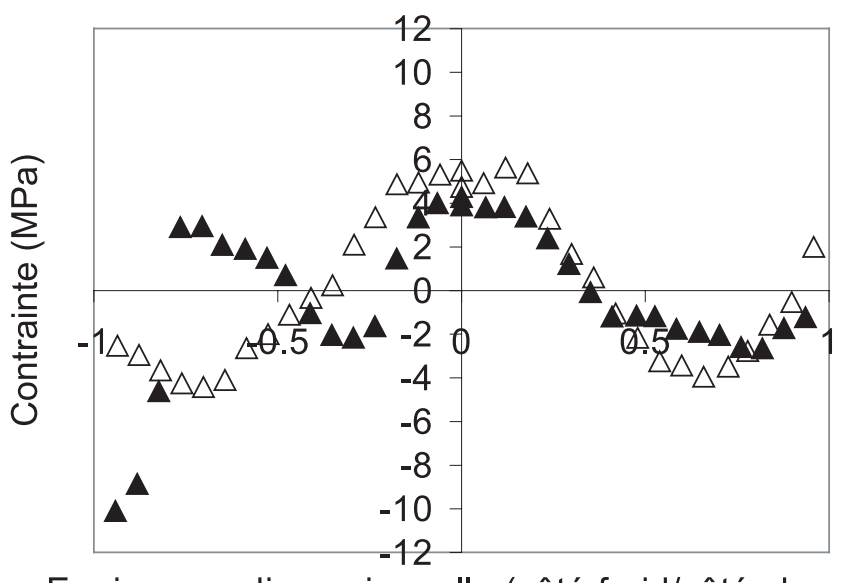

Epaisseur adimensionnelle (côté froid/côté chaud)

Fig. 11. Refroidissement dissymétrique pour le PAA 50 en positions $\mathrm{P} 1(\triangle)$ et $\mathrm{P} 2(\boldsymbol{\Lambda})$.

loin de l'entrée dans la cavité, l'effet de la pression de compactage est moins marqué, et on n'a plus de tension en surface. Le niveau de compression est marqué en peau du côté froid, en raison d'une vitesse de refroidissement plus élevée.

\section{Conclusions}

La genèse des contraintes et déformations résiduelles dans les pièces injectées en thermoplastique est maintenant bien décrite, avec la contribution des contraintes d'origine thermique, et de la pression figée. La présence de fibres de renfort entraîne une anisotropie et une hétérogénéité de comportement mécanique et de retrait. La méthode de mesure du moment fléchissant après enlèvement d'une fine couche de polymère permet de 
conduire au profil de contraintes dans des matériaux fortement anisotropes et hétérogènes comme les thermoplastiques renforcés. Nous avons montré cependant les difficultés pour obtenir un procédé d'enlèvement de couches non perturbateur. Nous avons appliqué cette technique à deux polyarylamides renforcés de 30 et $50 \%$ en poids de fibres de verre courtes. Les contraintes sont en général en traction au centre de l'éprouvette, et de compression vers les parois. Les paramètres du procédé ayant une incidence sur les contraintes sont la pression et le temps de maintien, et la température du moule. Il est délicat de préciser la façon dont les fibres agissent, puisque leur orientation variant dans l'épaisseur et dans le plan, les propriétés mécaniques, thermiques font de même.

Remerciements. Nous remercions MoldFlow France, Solvay, Schneider Electric pour leur aide technique et financière.

\section{Références}

[1] F.T.P. Baaijens, Calculation of residual stresses in injection moulded products, Rheol. Acta 30 (1991) 284-299

[2] N.J. Mills, Computation of residual stresses in extruded and injection moulded products, Plast. Rub. Proc. Applications 3 (1983) 181-188
[3] G. Titomanlio, V. Brucato, M.R. Kamal, Mechanism of cooling stress build-up in injection molding of thermoplastic polymers, Intern. Polym. Processing 1 (1987) $55-59$

[4] F. Boitout, J.F. Agassant, M. Vincent, Elastic calculation of residual stresses in injection moulding : influence of mold deformation and pressure in the liquid, Intern. Polym. Proc. 10 (1995) 237-242

[5] K.M.B. Jansen, Residual Stresses in Quenched and Injection Moulded Products, Intern. Polym. Processing 9 (1994) 82-89

[6] L.C.E. Struik, Internal stresses, dimensional stability and molecular orientation in plastics, John Wiley and Sons, Brisbane, 1990

[7] M. Vincent, Modelling of short fibre-reinforced thermoplastics flows in polymer processing, in : A.M. Cunha, S. Fakirov (ed.), Kluwer Academic Publishers, The Netherlands, 2000, pp. 345-363

[8] R.G. Treuting, W.T. Read, A mechanical determination of biaxial residual stress in sheet materials, J. Appl. Phys. 22 (1951) 130-134

[9] T. Giroud, Mesure et calcul des contraintes résiduelles dans les pièces injectées en thermoplastiques avec et sans fibres de renfort, Thèse de Doctorat, École des mines de Paris, 2001

[10] C. Eberhardt, A. Clarke, M. Vincent, T. Giroud, S. Flouret, Fibre orientation measurements in short glass fibre composites II. A quantitative error estimate of the 2D Image Analysis Technique, Comp. Sci. Tech. 61 (2001) 1961-1974 